\title{
Journal of Anesthesia and Surgery \\ Case Report \\ Primary Cesarean Delivery Complicated by the Incidental Finding of a Cerebral Aneurysm: Selecting a Safe Anesthesia Technique
}

\section{Won Chee}

Department of Anesthesiology, Montefiore Medical Center, Albert Einstein College of Medicine, NY, USA.

\section{Received date: December 28, 2014 \\ Accepted date: March 05, 2015 \\ Published date: March 09, 2015}

\begin{abstract}
*Corresponding author: Won Chee, M.D, Assistant Professor, Department of Anesthesiology, Montefiore Medical Center Albert Einstein College of Medicine, Bronx, NY, 10467 USA; Tel: 718920 4316; Fax: 718881 2245; E-mail:wchee@montefiore.org
\end{abstract}

Citation: Won Chee. Primary Cesarean Delivery Complicated by the Incidental Finding of a Cerebral Aneurysm: Selecting a Safe Anesthesia Technique (2015) J Anesth Surg 2(1) : 32-33.

\section{Introduction}

Possible diagnoses for headache during pregnancy include subarachnoid hemorrhage $(\mathrm{SAH})$ from cerebral aneurysm rupture. The incidence of SAH is 5-7 per 100,000 person-years for women between the ages of $15-44$ years $^{[1]}$. The incidental finding of a cerebral aneurysm in pregnancy raises a serious concern with possible rupture and an intracranial hemorrhage during vaginal or cesarean delivery. The case report considers the available anesthesia techniques in managing the patient safely. The written patient consent was obtained for publication.

\section{Case Presentation}

A 39 year-old obese female at 31 weeks gestation was scheduled for primary cesarean delivery. Her past medical history was significant for hypertension and chronic pain and weakness of her right arm and leg from a motor vehicle accident (MVA) 2 years ago. In addition, she developed wheezing at 24 weeks gestation and was newly diagnosed with asthma. Her past surgical history included appendectomy under general anesthesia. The patient was allergic to latex manifested by skin rash. Her current medications included acetaminophen, clindamycin, famotidine and subcutaneous heparin for DVT prophylaxis. Physical exam findings included: Height: $160 \mathrm{~cm}$, Weight: $94 \mathrm{~kg}, \mathrm{BMI}$ 36.7, BP 108/62, PR 103, RR 18, RA SpO $100 \%$. Her airway classification was Mallampati 3. The lungs were clear to auscultation but with decreased breath sounds at the base bilaterally. The heart rhythm was regular without murmur or gallop.

Since the MVA the patient had required multiple radiologic studies for chronic pain in her head, abdomen, right arm and right knee. All studies had yielded negative findings. As the patient complained of headache during this admission, an MRI of head was performed which revealed a 4-mm cerebral

\begin{abstract}
The presence of a cerebral aneurysm in an obstetric patient raises a special anesthetic concern for cesarean delivery. General anesthesia poses the inherently increased risk of a difficult airway as well as an adverse impact of hypertension during induction and emergence. Epidural anesthesia may facilitate gradual hemodynamic changes and allows intraoperative neurologic monitoring. However, potential dural puncture headache may lender it difficult to recognize an intracranial hemorrhage. In the end, spinal anesthesia was chosen for the advantage of avoiding hypertensive responses from either airway stimulation or inadequate analgesia.
\end{abstract}

aneurysm arising from the supraclinoid segment of the left internal carotid artery. Neurology consultation and the radiologic evaluation did not find any intracerebral hemorrhage or rupture. The obstetrician decided to proceed with cesarean delivery mainly to avoid hypertensive responses from prolonged labor and straining.

The patient was brought to the operating room and placed in a sitting position. The ASA standard monitors were placed; no invasive arterial line monitor was placed as the patient's blood pressure had been stable throughout the course of pregnancy. After prehydration ( $>2$ liters of Ringer's Lactate) spinal anesthesia was performed using a 25-gage Sprotte needle. T8 level block was achieved from the mixture of $1.5 \mathrm{ml}$ of $0.75 \%$ bupivacaine in $8.25 \%$ dextrose and $20 \mathrm{mcg}$ of fentanyl. The cesarean section proceeded uneventfully with the estimated blood loss of $800 \mathrm{ml}$. The Apgar score was 9/9. In short, the patient did not experience any anesthetic sequelae including spinal headache or intracranial hemorrhage. Nor did the patient develop any signs and symptoms of SAH postoperatively.

\section{Discussion}

In planning for cesarean delivery all available anesthesia techniques should be weighed their inherent complications associated with each technique against the risk of rupturing the aneurysm. According to the literature reviews, the actual risk for rupture and subarachnoid hemorrhage ( $\mathrm{SAH})$ is increased by various factors including the large size, advanced gestational age, African race, cigarette smoking, anticoagulation, and systemic hypertension ${ }^{[1,2]}$. Particularly, the role of systemic blood pressure was shown to be important in rupturing the aneurysm. In an animal study reduction of blood pressure with hydralazine

Copy rights: (C2015 Won Chee. This is an Open access article distributed under the terms of Creative Commons Attribution 4.0 
correlated well with prevention of aneurysm rupture ${ }^{[3]}$. Other risk factors are history of previous aneurysm rupture and a blunt head trauma with subsequent development of cerebral aneurysms $^{[4,5]}$. Her predispositions for a cerebral aneurysm included hypertension and possibly the head trauma from the MVA.

It is strongly speculated that pregnancy increases the rate of rupture from physiologic and hormonal changes ${ }^{[6]}$. Yet, studies have not established pregnancy and delivery increase the risk of aneurysm rupture with the exception of a slight increase in 6 weeks after delivery ${ }^{[7]}$. According to a review of cases from 1988 to 2009 , the risks of rupture during pregnancy and deliveries were $1.4 \%$ (against $1.8 \%$ prevalence of unruptured aneurysms among women of the fertile age group ${ }^{[8]}$. Labor does not seem to increase the risk of rupture either. Nor is there any difference between cesarean and vaginal delivery with respect to the $\operatorname{risk}^{[9]}$.

In regards to the management of anesthesia, few case reports found in the literature review generally chose regional anesthesia over general anesthesia as long as the mother's neurological status was stable. Sharma, et al. reported cesarean delivery successfully performed under the continuous epidural technique. Postoperatively, epidural anesthesia was continued to facilitate adequate analgesia and stable hemodynamics ${ }^{[10]}$. It is important to note the advantage of the continuous regional technique considering the risk of aneurysm rupture remains immediately after the delivery. Also, Handa, et al. in Japan reported three cases of Cesarean deliveries for the patients with cerebral aneurysm rupture. Two were performed under general anesthesia for the mothers with neurologic symptoms from bleeding while one case was done under spinal anesthesia for the patient whose neurologic condition was normal ${ }^{[11]}$. The preference appears to be the result of anesthetic concerns associated with pregnancy in general and the cerebral aneurysm in specific, namely, failed intubation and the hypertensive response. The maternal mortality associated with anesthesia is significantly greater (16.7 times) under general anesthesia than regional anesthesia ${ }^{[12]}$. As a result, the majority of the cesarean deliveries in the U.S. are performed under a regional technique. The main cause of the maternal death from anesthesia is the result of failed intubation and hypoventilation as the incidences of airway edema and difficult intubation increase by 10 fold during pregnancy. Nevertheless, general anesthesia may be necessary if the mother's mental status is compromised from intracerebral hemorrhage and unable to protect her airway against aspiration.

We first considered general anesthesia for the advantages in case of aneurysm rupture and SAH hemorrhage. However, we decided against it from the immediate concern of a difficult airway along with obesity and asthma. Also, airway stimulation would have elicited hypertensive responses during induction and emergence. Next, we considered the epidural anesthesia technique. Epidural anesthesia facilitates gradual hemodynamic changes and allows intraoperative neurologic monitoring. However, potential headache from dural puncture with a large bore needle was confounding with elucidating SAH. We did not want any difficulty in recognizing aneurysm rupture during the procedure. Ultimately, we selected the spinal anesthesia technique for more reliable block so that we avoid hypertension from intubation or inadequate analgesia.

\section{Conclusion}

In summary, a realistic and balanced perspective is important in choosing the appropriate anesthesia technique for cesarean delivery. Not only the risk of a SAH hemorrhage but also all other risk factors associated with anesthetic mortality and morbidity should be weighed carefully in each case.

Conflict of Interest: I, Won Chee, declare that I do not have any conflict of interests regarding the manuscript.

\section{References}

1. Bateman, B.T., Schumacher, H.C., Bushnell, C.D., et al. Intracerebral hemorrhage in pregnancy: frequency, risk factors, and outcome. (2006) Neurology 67(3): 424-429.

2. Juvela, S., Porras, M., Poussa, K. Natural history of unruptured intracranial aneurysms: probability of and risk factors for aneurysm rupture. (2008) J Neurosurg 108(5): 1052-1060.

3. Tada, Y., Wada, K., Shimada, K., et al. Roles of hypertension in the rupture of intracranial aneurysms. (2014) Stroke 45(2): 579-586.

4. Unruptured intracranial aneurysms--risk of rupture and risks of surgical intervention. International Study of Unruptured Intracranial Aneurysms Investigators. (1998) N Engl J Med 339(24): 1725-1733.

5. Dubey, A., Sung, W.S., Chen, Y.Y., et al. Traumatic intracranial aneurysm: a brief review. (2008) J Clin Neurosci 15(6): 609-612.

6. Mas, J.L., Lamy, C. Stroke in pregnancy and the puerperium. (1998) J Neurol 245(6-7): 305-313.

7. Kittner, S.J., Stern, B.J., Feeser, B.R., et al. Pregnancy and the risk of Stroke. (1996) N Engl J Med 335: 768-774.

8. Kim, Y.W., Neal, D., Hoh, B.L. Cerebral aneurysms in pregnancy and delivery: pregnancy and delivery do not increase the risk of aneurysm rupture. (2013) Neurosurgery 72(2): 143-149.

9. Dias, M.S., Sekhar, L.N. Intracranial hemorrhage from aneurysms and arteriovenous malformations during pregnancy and the puerperium. (1990) Neurosurgery 27(6): 855-867.

10. Sharma, S.K., Herrera, E.R., Sidawi, J.E., et al. The pregnant patient with an intracranial arteriovenous malformation. Cesarean or vaginal delivery using regional or general anesthesia? (1995) Reg Anesth 20(5): 455-458.

11. Handa, F., Tanaka, M., Toyooka, H. Anesthetic management of parturients with intracranial arteriovenous malformation. (1997) Masui 46(8): 1110-1113.

12. Hawkins, J.L., Koonin, L.M., Palmer, S.K., et al. Anesthesia-related deaths during obstetric delivery in the United States, 1979-1990. (1999) Anesthesiology 86(2): 277- 284. 